(Aus dem physiologischen Institut der Universität Marburg.)

\title{
Über das für die Lungenvaguswirkung neutrale Lungrenvolum.
}

Von

Dr. M. Ishihara, Fukwoka (Japan).

(Mit 11 Textfiguren.)

Nach den neueren Untersuchungen von Professor F. Sch enck ${ }^{1}$ ) werden bei der gewöhnlichen ruhigen Exspiration nicht nur die inspiratorisch wirksamen Vagusfasern nicht erregt, sondern es hört noch nicht einmal die Erregung der exspiratorisch wirksamen Fasern ganz auf; die inspiratorisch wirksamen Fasern werden erst bei starkem Lungenkollaps, wie man ihn durch starke künstliche Aussaugung der Lungen erhält, erregt. Es muss daher für die natürliche Erregung des Lungenvagus eine neutrale Stellung der Lungen geben, in der weder die inspiratorische noch die exspiratorische Wirkung des Vagus auf die Atmung zustande kommt, und zwar muss diese Stellung bei einem kleineren Lungenvolum vorhanden sein als bei dem Volum, das die Lunge in gewöhnlicher ruhiger Exspirationsstellung hat. Auf Veranlassung von Herrn Professor F. Schenck habe ich einige Untersuchungen über diese Neutralstellung angestellt, über deren Resultate ich im folgenden berichten will.

I.

Zunächst habe ich an fünfzehn schwach chloralisierten Kaninchen nach dieser Neutralstellung gesucht. Das Versuchstier wurde tracheotomiert, und die in die Trachea eingelegte Kanüle wurde aussen mit dem einen Schenkel einer H-förmigen Glasröhre luftdicht verbunden. An den zweiten Schenkel des H-Rohres war ein durch Klemmpinzette verschliessbares kurzes Gummischlauchstück angesetzt; der dritte

1) Dieses Arch. Bd. 100 S. 337. 1903. 
Schenkel wurde durch einen dickwandigen Gummischlauch mit einem Gummimanometer und der vierte mit einer Saugvorrichtung verbunden; letztere bestand aus zwei grossen, unten tubulierten Flaschen; die Tubuli der beiden Flaschen waren durch einen Gummischlauch verbunden, die Flaschen zur Hälfte mit Wasser gefüllt. In den Hals der einen Flasehe war ein durchbohrter Gummipfropf eingesetzt, und in der Durchbohrung des Pfropfens steckte ein Glasrohr, das mit jenem vierten Schenkel des H-Rohres dureh den einen der oben erwähnten dickwandigen Gummischläuche verbunden wurde. Wurde die andere Flasche tiefer als die erste gestellt, so floss das Wasser in sie über und saugte Luft aus der Röhrenleitung zwischen der Trachealkanüle und dem Manometer heraus. Damit das Aussaugen plötzlich geschehen konnte, wurde vor dem Tieferstellen der einen Flasche an den Schlauch zwisehen H-Rohr und Aussaugvorrichtung noch eine Klemmpinzette angelegt, die nach Tieferstellen der Flasche in dem Momente, wo ausgesaugt werden sollte, gelöst, gleich danach aber wieder angelegt wurde; die Lunge blieb danach in dem ausgesaugten Zustande, bis die zuerst erwähnte Klemmpinzette an dem kurzen Gummischlauchstück gelöst und dadurch die Kommunikation der Luftwege nach aussen wieder freigegèben war. Auch das Aufblähen der Lungen wurde in den später zu : be. schreibenden Versuchen mit dieser Vorrichtung bewirkt, indeñ die zweite Flasche höher gestellt wurde als die erste.

Die beiden Nervi vagi wurden am Halse des Versuchstieres freigelegt; der eine Vagus wurde durchschnitten, an den anderen Vagús wurden vermittelst der mit Kochsalzlösung getränkten: Wollfäden drei unpolarisierbare Elektroden angelegt in der: Weise, wie es $P f l u ̈ c k e r$ in der dieser vorangehenden Abhandlung beschrieben hat. Die Elektroden waren mit einer Akkumulatorenbatterie verknüpft; der elektrische Strom diente zur reizlosen Vagusausschaltung durch Anelektrotonus in der von Schenck und von Pflü cker beschriebenen Weise Die Spannung der angewandten Elemente der Batterie betrug bei den Versuchen 6 bis 12 Volt. Der Strom wurde immer so stark gewählt, dass die inspiratorische Wirkung, welche nach Vagusausschaltung bei Fixation der Lungen in gewöhnlicher Exspirationsstellung immer zu erhalten ist, bei Durchleitung des Stromes sicher zustande kam.

Der einzelne Versuch wurde nun in folgender Weise angestellt: Bei einer: gewöhnlichen Exspirationsstellung wird das vorher offene 
kurze Gummischlauchstück am H-Rohr durch die erste Klemmpinzette geschlossen, und gleich danaçh, während die nächste Inspiration im Gange ist, wird : die zweite Klemmpinzette an der Saugvorrichtung für kurze Zeit gelöst, dann wied"er bei ausgesaugtem Zustande der Lungen geschlossen; und nun werdèn die Atemanstrengungen des Tieres bei dieser Lungénistellung durch das Manometer auf die Kymographiontrommel aufgeschrieben ${ }^{\text {Nach }}$ einigen Sekunden wird die erste Klemmpinzette gelöst, das Tier kann ins Freie atmen, und es wird ihm einige Zeit Ruhe gegönnt. Es wird alsdann der Versuch nochmals in der gleichen Weise wiederholt; nur wird das zweite Mal sofort nach der Aussaugung, die um denselben Betrag wie das erste Mal erfolgt sein muss, auch der zur Vagusaussehaltung dienende Strom geschlossen: Solcher Versuchsreihen mit und ohne Vagusausschaltung werden mehrere nacheinander angestellt bei verschieden starker Aussaugung, die erreicht wurde dadurch, dass die zweite Flasche der Saugvorrichtung bald mehr, bald weniger tief unter die erste gestellt wurde.

Auf solche Weise konnte ich immer eine Stellung des Lungenvolums finden, bei der die Vagusausschaltung weder eine deutlich exspiratorische noch eine deutlich inspiratorische Wirkung hatte, während bei stärkerer oder schwächerer Aussaugung eine exspiratorische oder inspiratorische Wirkung hervortrat. Diese Neutralstellung fand ich an $1500-2000 \mathrm{~g}$ schweren Kaninchen bei einem Lungenvolum, das einem negativen Drucke von 15-30 mm Queeksilbersäule in der Aussaugvorrichtung entspricht. Es ist natürlich nicht möglich, das Lüngenvolum der Neutralstellung in absoluter Grösse anzugeben, und ich muss mich daher auf die Angabe beschränken, durch welchen negativen Druck das Volum erreicht wurde:

Als Beleg für meine Angaben dienen die Kurven der Fig. 1, 2 und 3. In diesen Figuren wie in allen folgenden sind die Kurven von links nach rechts zu lesen. Mit ein und derselben römischen Zahl ist immer je ein Paar Versuche mit gleichem Aussangungsgrade bezeichnet; $a$ gibt dabei den Versuch ohne, $b$ mit Vagusausschaltung an. Über der Kurve jedes Versucbes ist auch der Druck angegeben, bei dem die Aussaugung erfolgt war. In diesen wie in allen weiteren Kurven bedeutet immer die Senkung der Kurve Aussaugen resp.: Inspirationsanstrengung, die Hebung Aufblähen resp. Exspirationsanstrengung. Oberhalb der Atmungskurve ist die Schliessung und Öffnung des den Vagus ausschaltenden Stromes. 


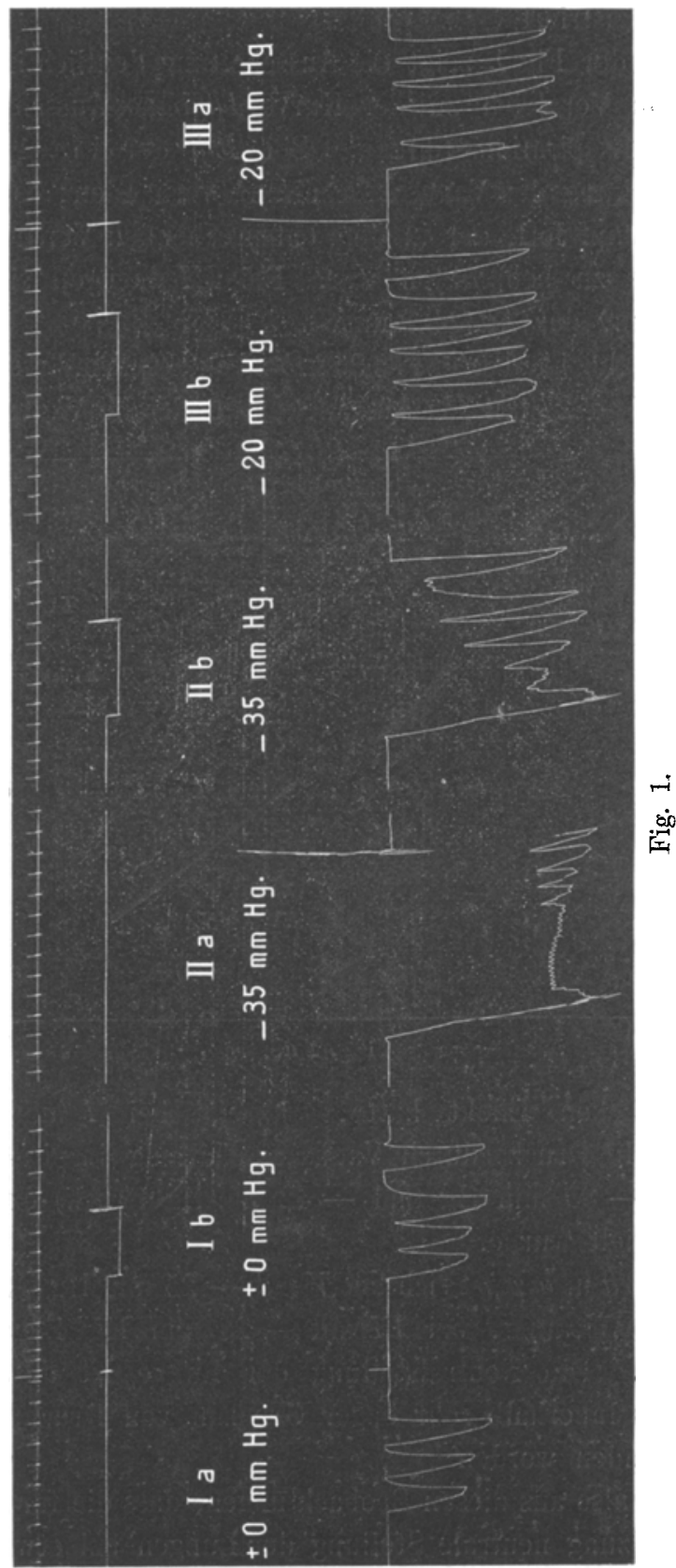


vermittelst eines elektrischen Signalschreibers markiert und die Zeit in Sekunden registriert.

Bei $1 b$ der Fig 1 zeigt die Ausschaltung deutlich inspiratorische Wirkung im Vergleich zu der Kurve $1 a$ ohne Ausschaltung in gewöhnlicher Exspirationsstellung. Bei $I I b$ der Fig. 1 ist exspiratorische Wirkung der Vagusausschaltung vorbanden; es fällt der Inspirationskrampf fort, der in dem entsprechenden Versuche IIa ohne Ausschaltung zu beobachten war. Bei $I I I$ dagegen sieht man fast keine Wirkung der Ausschaltung.

Ganz analog ist aus der Fig. $2 a$ u. $b$ zu entnehmen, dass im Versucbspaar III (bei $-27 \mathrm{~mm} \mathrm{Hg}$ Aussaugungsdruck) die Vagusausschaltung

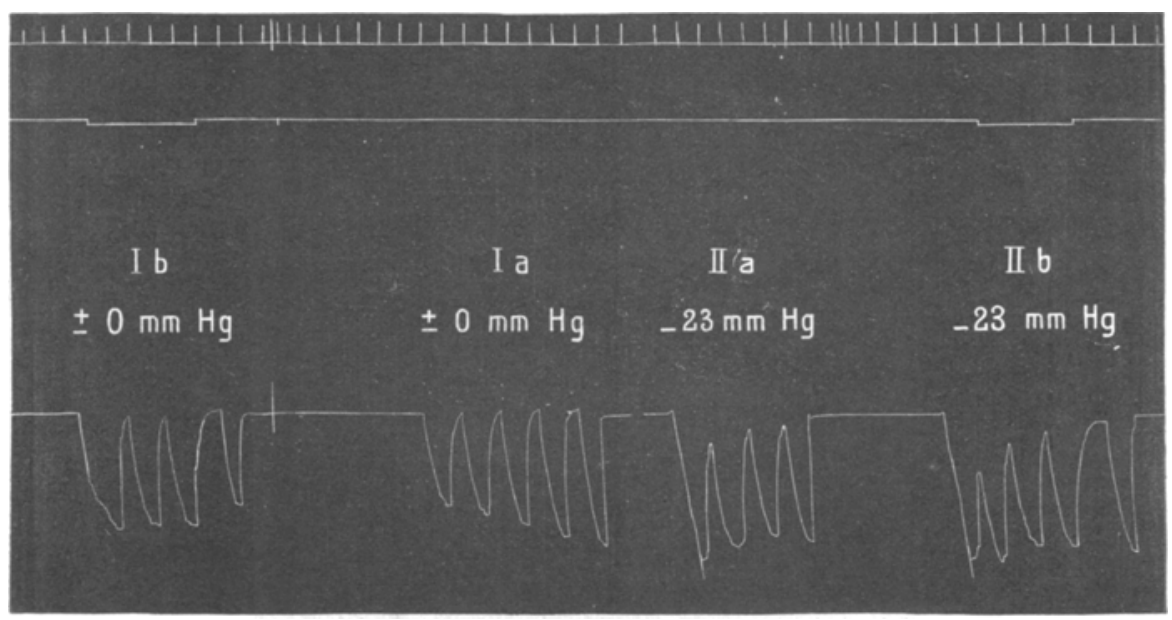

Fig. $2 a$.

keinen deutlichen Effekt hatte, während bei $I$ und $I L$ ( 0 und - $23 \mathrm{~mm} \mathrm{Hg}$ Druck) durch Vagusausschaltung inspiratorische, bei IV und $V(-35 \mathrm{~mm} \mathrm{Hg}$ und $-42 \mathrm{~mm} \mathrm{Hg}$ Druck) exspiratorische Wirkung erzielt wurde.

In Fig. $3 a$ u. $b$ zeigt sich bei $V$ mit $-25 \mathrm{~mm} \mathrm{Hg}$ Druck noch inspiratorische Wirkung, bei $V I$ mit $-28 \mathrm{~mm} \mathrm{Hg}$ Druck noch exspiratorische. Die Neutralstellung der Lunge ist also in diesem Versuche als innerhalb sebr enger Grenzen von Druckunterschieden liegend gefunden worden.

Es folgt also aus diesen Beobachtungen, dass die für die Wirkung der Vagusreizung neutrale Stellung der Lungen bei etwas kleinerem Volumen liegt, als der gewöhnlichen Exspirationsstellung entspricht. Der exspiratorische Effekt der Vagusausschaltung tritt um so stärker 


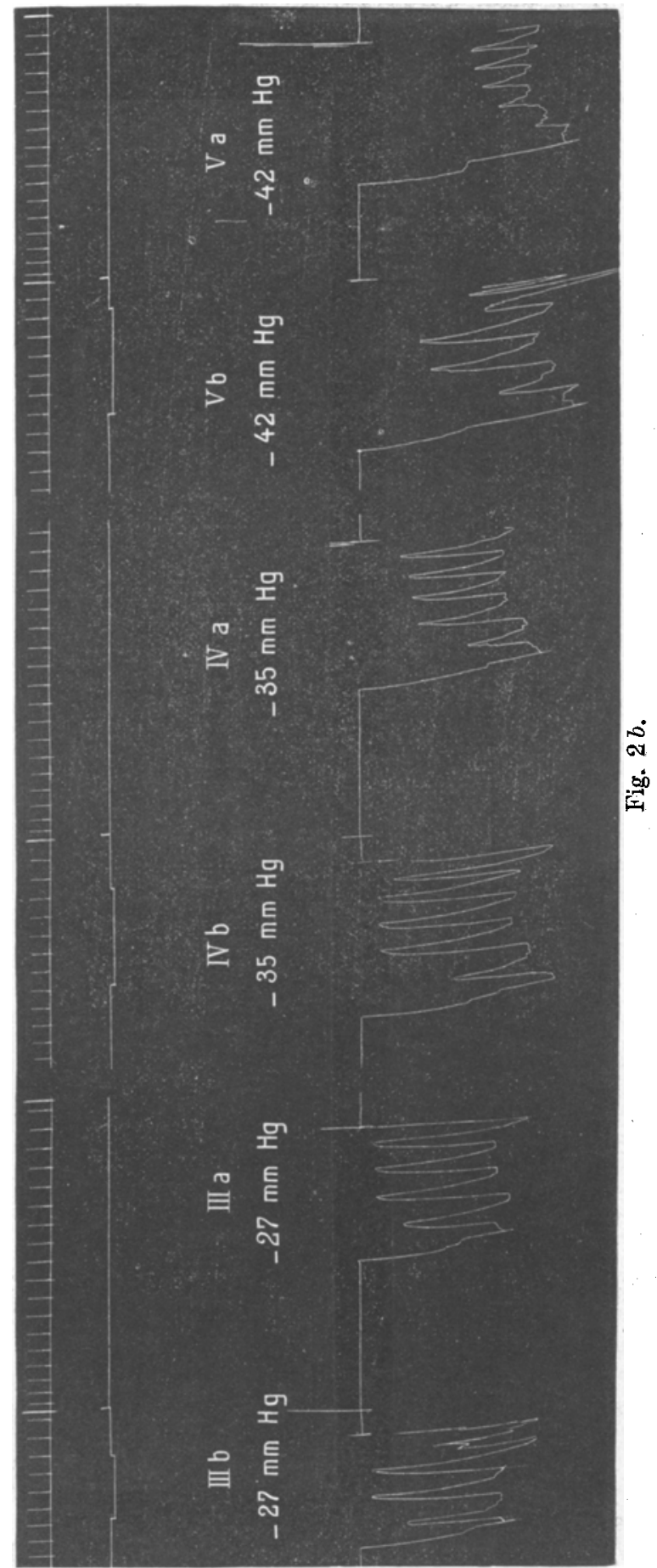



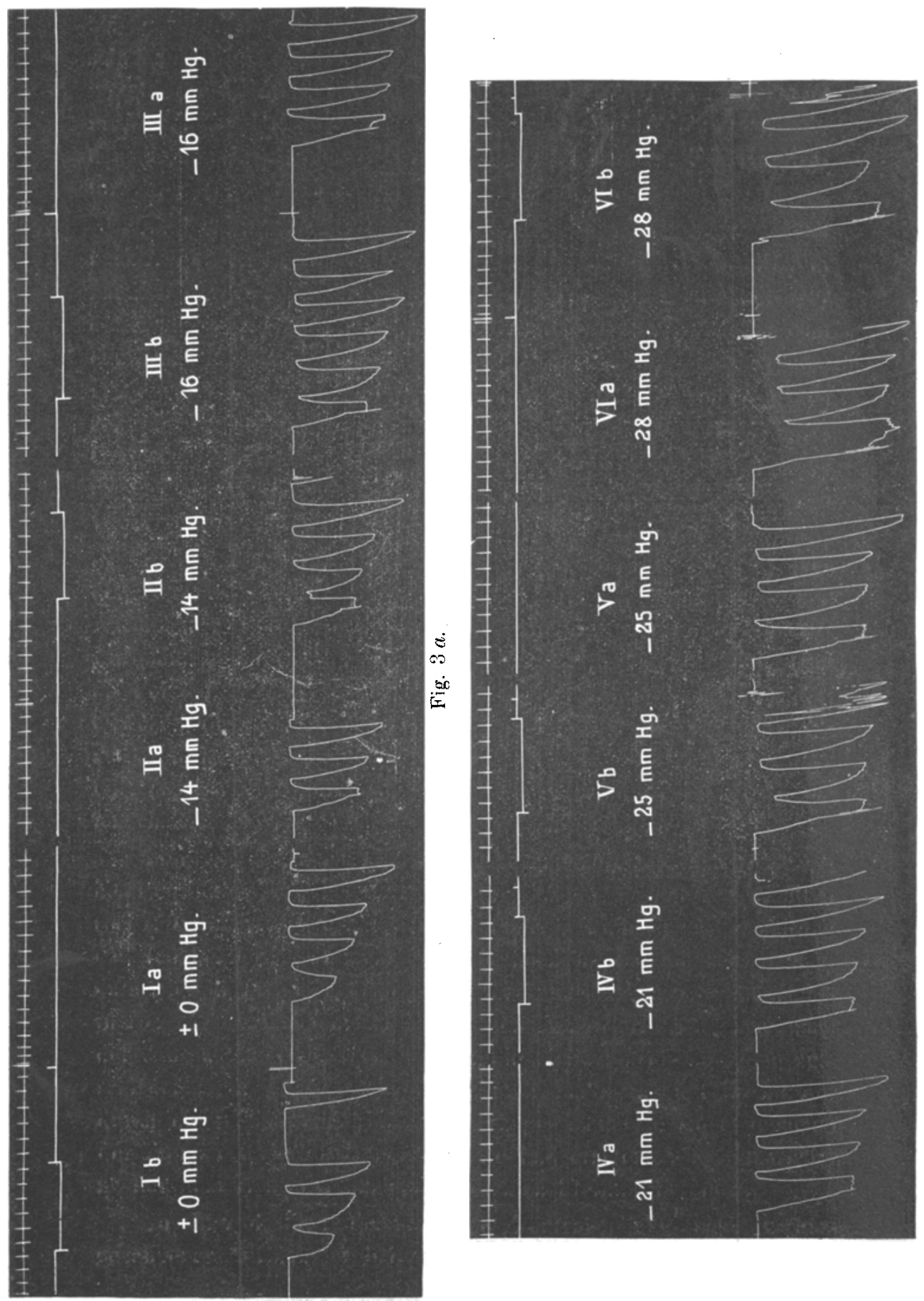
hervor, je kleiner, von dieser Neutralstellung aus gerechnet; das Lungenvolum wird; der inspiratorische Effekt der Vagusausschaltung tritt um so mehr hervor; je grösser das Volum, von dieser Neutralstéllung aus gerechnet; ist.

\section{II.}

$\because$, Es fragt sich nun, ob die Neutralstellung unter allen Umständen immer bei demselben Lungenvolum bleibt; oder ob sie wechseln kann.

Um auf diese Frage experimentell eine Antwort geben zu können, habe ich : untersucht, ob etwa durch ungewöhnlich lang ausgedehnte natürliche Erregung der einen oder anderen Lungenfaserart eine Veränderung der Lage der Neutralstellung hervorzubringen ist. Die Reizung der exspiratorisch wirksamen Fasern dureh Aufblähunig dèr Lungen wurde hierzu als das bequemste Mittel verwandt. Die Versuchsanordnung ist dieselbe wie oben erwähnt; nur wurde hier aufgebläht statt ausgesaugt. Gleich nach dem Zuklemmen des kurzen Gummischlauchstückes blähte ich durch kurzes Öffnen der zweiten Klemmpinzette an der ersten Flasche, welche hier niedriger gestellt ist als die andere, die Lungen anf und fixierte sie so einige Zeit lang in aufgeblähtem Zustande. Kurz bevor nach dem dadureh bewirkten exspiratorischem Stillstande noch die erste Inspiration wieder zu erwarten war, brachte ich durch ein kurzes Öffnen der Klemmpinzette am kurzen Gummischlauchstück die Lungen wieder zum gewöhnlichen Lungenvolum in Exspirationstellung zurück und registrierte die Atemanstrengungen bei dem so fixiert gehaltenen: Lungenvolum.. In dieser Art wurden je zwei Versuche angestellt, der erste ohne, der zweite mit Vagusausschaltung während der Atemanstrengung in Exspirationsstellung; im zweiten Falle wurde der Vagus ausgeschaltet, sobald die Lunge wieder die gewöhnliche Exspirationsstellung hatte. In den verschiedenen Versuchspaaren wurde die Aufblähung verschieden stark gemacht; daneben wurde wiederbolt die Lunge ohne vorausgegangene Aufblähung in gewöhnlicher Exspirationsstellung mit und ohne Vagusausschaltung fixiert und die Atemanstrengung registriert.

Bei diesem Versuchsplane war ich von der Erwägung ausgegangen, dass durch die langdauernde Erregung der exspiratorisch wirksamen Lungenvagusfasern diese weniger wirksam, die inspiratorisch wirksamen dagegen wirksamer geworden sein konnten, so dass letztere jetzt schon bei grösserem Lungenvolúm, vielleicht schon 
bei gewöhnlicher Exspirationsstellung stärker wirksam geworden sein konnten als erstere. Wenn diese Überlegung richtig wäre, dann müsste der Effekt der Vagusausschaltung auf die Atemanstrengung in Exspirationsstellung nach vorausgegangener Aufblähung gerade umgekehrt sein als ohne die Aufblähung: im letzteren Falle inspiratorisch, im ersteren Falle dagegen exspiratorisch.

Eine solche Veränderung der Wirkung der Vagusausschaltung nach Aufblähen konnte ich tatsächlich in einigen Fällen konstatieren, nämlich einen exspiratorischen Effekt durch Vagusausschaltung oder doch wenigstens keinen inspiratorischen Effekt mehr, während ein deutlicher Inspirationseffekt bei Vagusausschaltung ohne vorausgegangene Aufblähung erhalten war. Als Beleg für diese Angaben gebe ich in den Fig. 4 und 5 Beispiele von solchen Kurven. Der Grad der Aufblähung ist hier auch in Millimeter Quecksilbersäule angegeben; im übrigen sind die Kurven nach dem früher Gesagten leicht verständlich.

In Fig. 4 sieht man bei $I b$ zunächst den inspiratorischen Effekt der Vagusausschaltung in Exspirationsstellung ohne vorausgegangene Aufblähung. Bei $I I a$ und $b$ sind die Versuche mit vorausgegangener Aufblähung dargestellt; bei II a ohne Vagusausschaltung sieht man als Folge der vorausgegangenen Aufblähung einen längeren inspiratorischen Stillstand; bei $I I b$ hält dieser Stillstand infolge Vagusausschaltung nicht so lange an; die erste Exspiration tritt früher ein; das ist der Ausdruck einer exspiratorischen Wirkung der Vagusausschaltung.

In Fig. 5 ist auch ohne vorausgegangene Aufblähung eine erhebliche inspiratorische Wirkung durch Vagusausschaltung erhalten (bei $I I b$ ), mit vorausgegangener Aufblähung hat die Vagusausschaltung dagegen keinen Effekt mehr (vgl. $I b$ und $I a)$. Beilüufig sei bemerkt, dass hier in $I a$ nicht etwa die Aufblähung stärker gewesen ist als in $I b$; wie das bei einem Blick auf den Teil der Kurven gleich nach der Aufblähung erscheinen könnte; die hier zu beobachtenden Verschiedenheiten waren vielmehr dadurch bedingt, dass in $I a$ der durch die plötzliche Aufblähung stark geschleuderte Hebel oben an der Trommelfäche etwas gehakt hatte und erst etwas später wieder zu der dem Lungenvolum èntsprechẹnden Stellung herabfiel.

In Fig. 4 liegt demnach die Neutralstellung nach vorausgegangener Aufblähung bei etwas grösserem Lungenvolum, als der 
Über das für die Lungenvaguswirkung neutrale Lungenvolum.

gewöhnlichen Exspirationsstellung entspricht, in Fig. 5 bei dem Volum der gewöhnlichen Exspirationsstellung selbst.

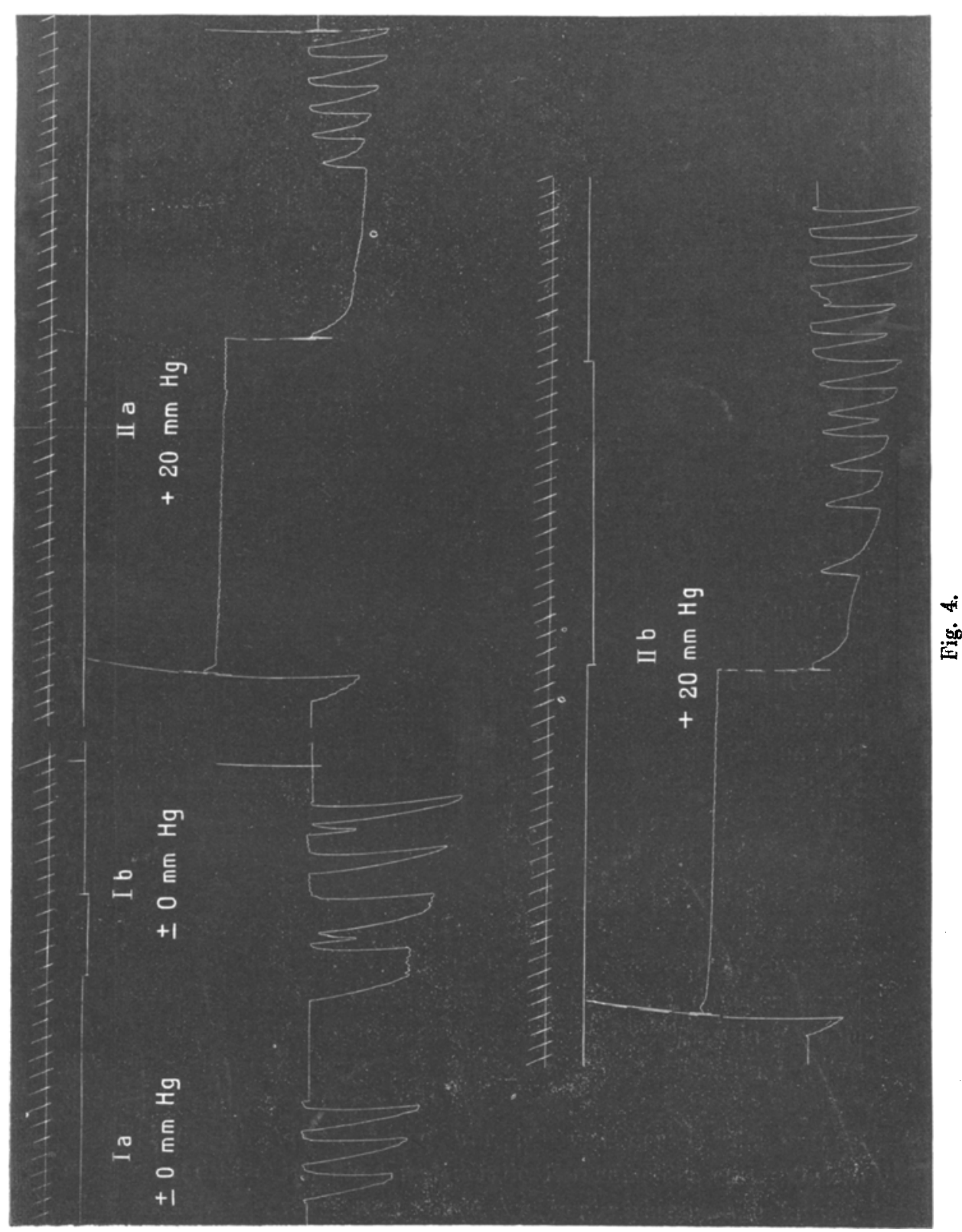

Dies Versuchsresultat kam allerdings in meinen an zahlreichen Versuchstieren angestellten Versuchen nur sehr selten vor. In den 
M. Ishihara:

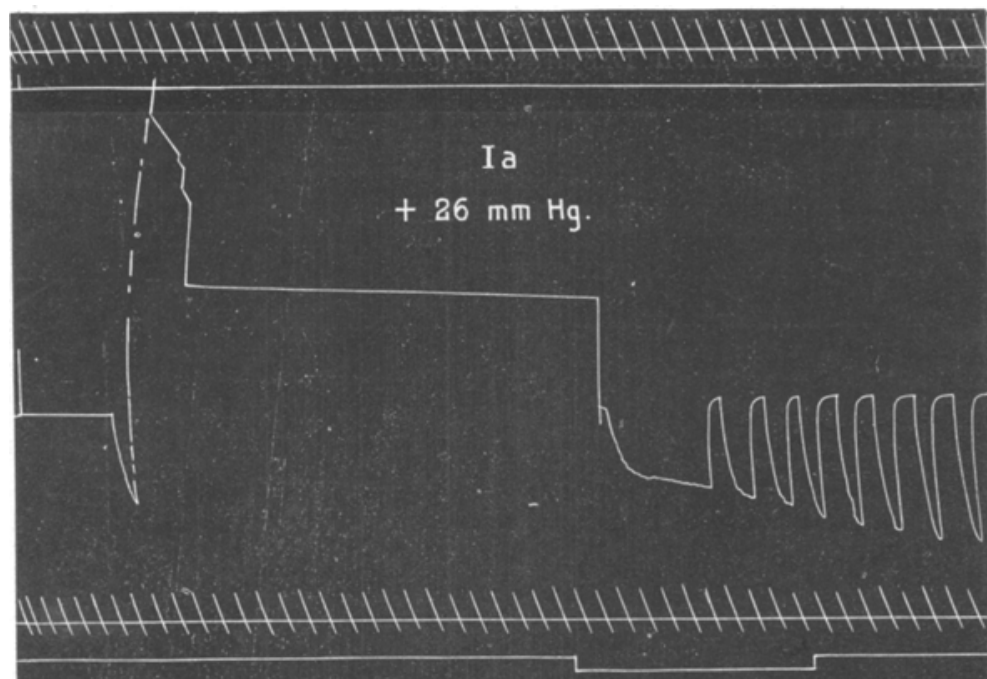

I b

$+26 \mathrm{~mm} \mathrm{Hg}$.
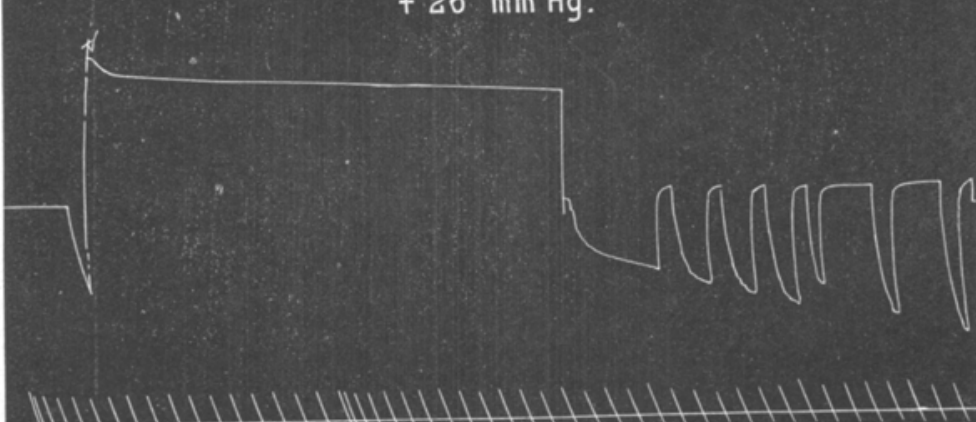

II a

$\pm 0 \mathrm{~mm} \mathrm{Hg}$.
II 6

$\pm 0 \mathrm{~mm} \mathrm{Hg}$.

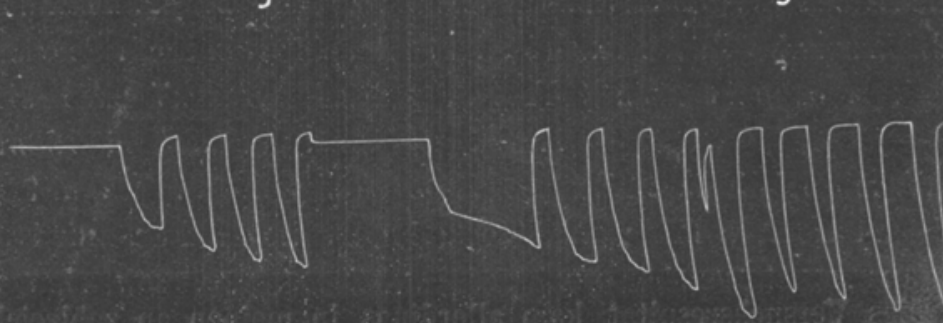

Fig. 5 . 


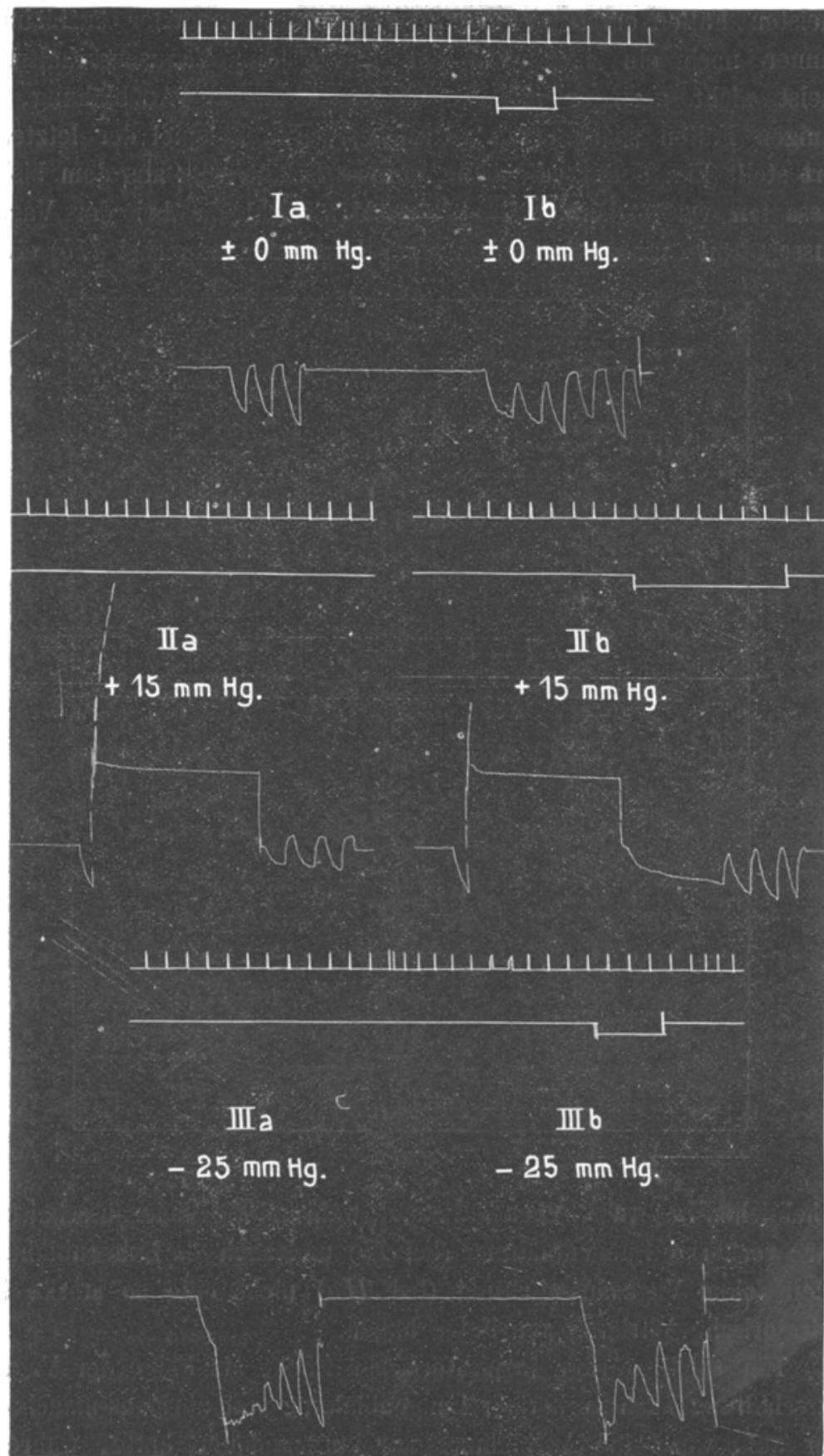

Fig. 6 . 
meisten Fällen ergab sich auch nach vorausgegangener Aufblähung immer noch ein inspiratorischer Effekt der Vagusausschaltung, meist nicht geringer als ohne vorausgegangene Aufblähung, in einigen Fällen sogar etwas deutlicher. Ein Beispiel der letzteren Art stellt Fig. 6 dar, die ohne weitere Erläuterung aus dem bisher Gesagten verständlich sein wird. Man sieht da bei der Vagusausschaltung nach vorausgegangener Lungenblähung (bei $I I b$ ) einen

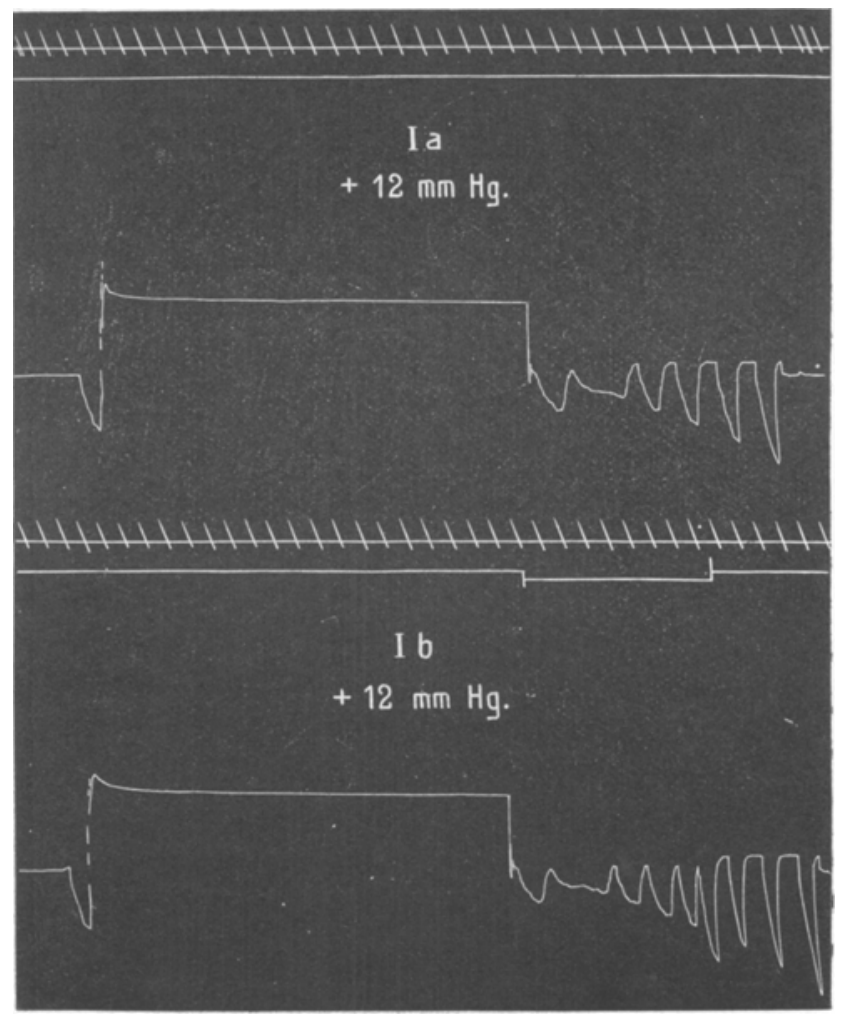

Fig. $7 a$.

erheblichen inspiratorisehen Stillstand, während die Vagusausschaltung ohne vorherige Lungenblähung (bei $I b$ ) und auch die Lungenblähung allein ohne Vagusausschaltung (bei $I I a$ ) nicht eine so starke inspiratorische Wirkung zur Folge haben.

Ein solch starker inspiratorischer Effekt als Folge der Vagusausschaltung nach vorheriger Lungenblähung trat ubrigens manchmal nur auf, wenn die Aufblähung nur kurz gedauert hatte, während nach längerer Aufblähung dieser inspiratorische Effekt wieder 
schwächer oder gar unmerklich wurde. Ein Beispiel dieser Art gibt die Fig. $7 a$ u. $7 b$, wo ohne vorherige Aufblähung der inspiratorische Effekt der Vagusausschaltung nur gering war (bei $I I b$ ); nach kurzer Aufblähung bei $I I I b$ tritt dieser Effekt viel stärker hervor, um nach längerdauernder Aufblähung (bei $I b$ ) wieder unmerklich zu werden.

Über die Ursache dieser verstärkten inspiratorischen Wirkung der Vagusausschaltung lässt sich auf Grund der bisher beschriebenen

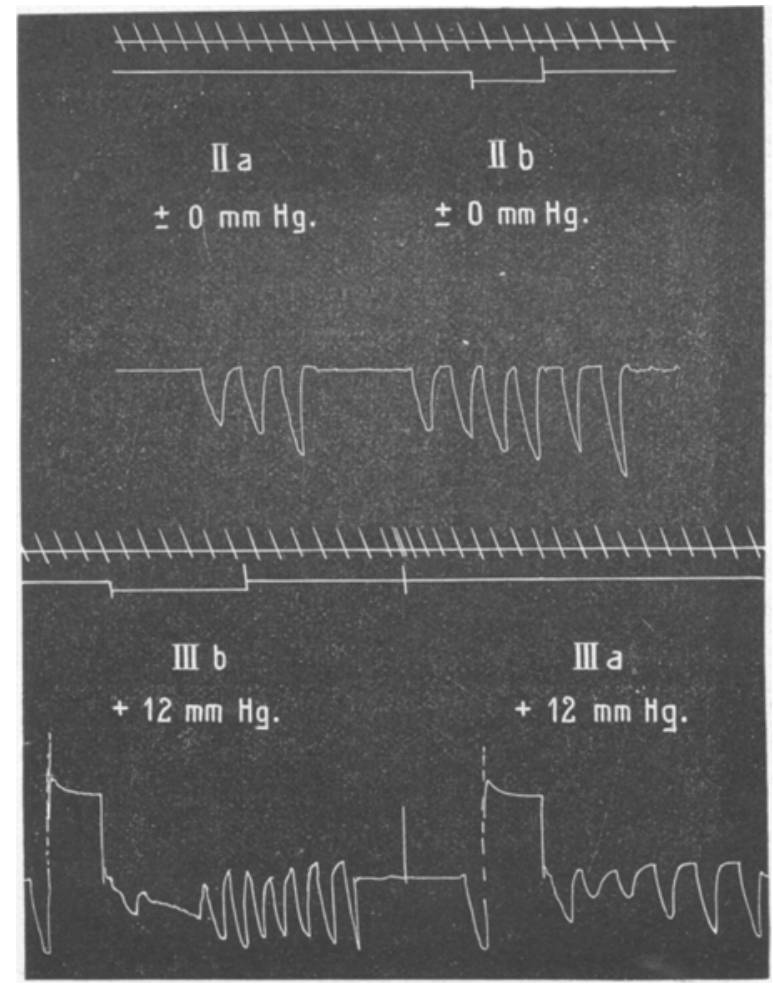

Fig. $7 b$.

Versuche noch nichts Sicheres aussagen. Möglich wäre es, dass sie beruht auf einer Verlagerung der Neutralstellung der Lungen nach unten hin, d. h. die Neutralstellung läge bei kleinerem Lungenvolum als in der Norm; jedoch sind auch andere Erklärungen zulässig.

Um etwas mehr Sicherheit in der Frage der Verlagerung der Neutralstellung zu gewinnen, wurden daher schliesslich noch Versuche angestellt, in denen die Lungen zwar zuerst auch aufgebläht, danach aber nicht bloss bis zu gewöhnlicher Exspirationsstellung 
wieder zurückgeführt, sondern durch Aussaugen noch mehr verkleinert wurden, etwa bis zu dem Volumen, das der normalen Neutralstellung entsprach. Es wurde dann der Effekt der Vagusausschaliung bei dieser Stellung verglichen mit der Wirkung, die die.Vagus̉ausschaltung

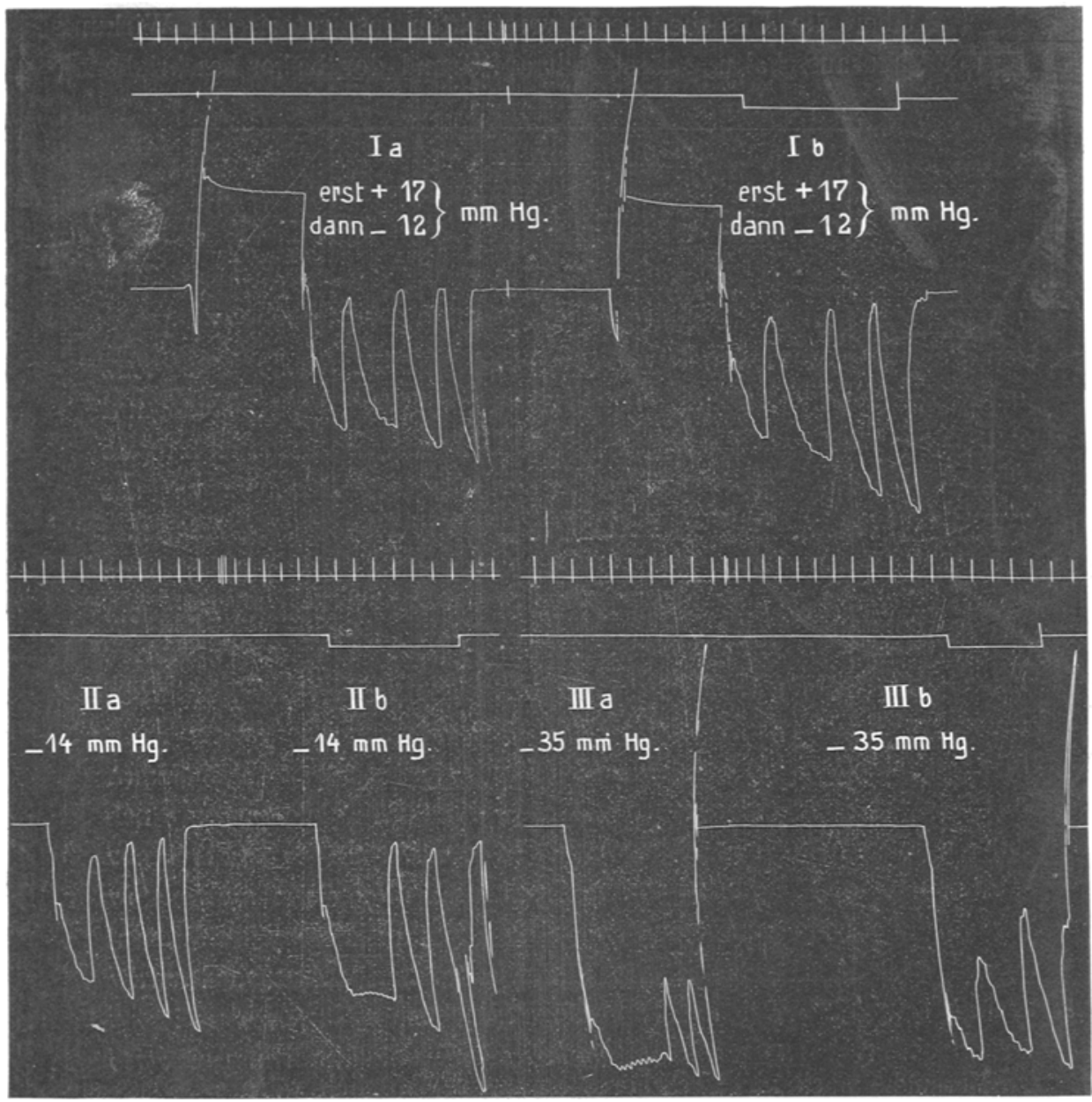

Fig. 8.

hatte, wenn diese Stellung ein zweites Mal durch Aussaugen allein, ohne vorberige Aufblähung, erreicht war. Diese Versuche haben ausnahmslos: ergeben, dass dureh die Aufblähung niemals eine Verlagerung 3 , der Neutralstellung zu einem kleineren Lungenvölum stattgefunden hatte; sondern dass danach entweder die Neutral- 
stellung bei demselben oder bei einem etwas grösseren Lungenvolum zu finden war.

Ein Beispiel der letzteren Art wird durch die Fig. 8 gegen. Hier ist ohne vorherige Aufblähung bei Aussaugung mit $-14 \mathrm{~mm}$. Quecksilberdruck noch inspiratorische Wirkung der Vagusausschaltung vorbanden (siehe $I I b$ ), bei Aussaugung mit - $35 \mathrm{~mm}$ Quecksilberdruck dagegen exspiratorische Wirkung (siehe III b). Als nun erst eine nicht sehr lange Aufblähung bewirkt worden war und danach Aussaugen mit - $12 \mathrm{~mm}$ Druck, hatte die Vagusausschaltung nur noch eine Spur von inspiratorischer Wirkung; es war jetzt also bei - $12 \mathrm{~mm}$ Aussaugungsdruck fast die Neutralstellung erreicht, während sie ohne vorherige Aufblähung bei $-14 \mathrm{~mm}$ Aussaugungsdruck noch nicht erreicht war.

Im ganzen führen meine Versuchsresultate $\mathrm{zu}$ dem Schlusse, dass durch Aufb]ähen der Lungen eine Verlagerung der Neutralstellung zu einem grösseren Lungenvolum bewirkt werden kann, dass aber diese Verlagerung nur um einen geringen Betrag erfolgt und überhaupt nicht leicht zu erzielen ist; meist bleibt die Lage der Neutralstellung unverändert.

Was nun die Ursache der Verlagerung der Neutralstellung anlangt, so lassen sich darüber vorläufig nur Vermutungen aussprechen; ich unterlasse es daher, hier darauf einzugehen; es wird darüber:an anderer Stelle von Herrn Professor S chenck mehr gesagt werden. 